\title{
The Arts Health Early Career Research Network: Link, Learn, Lead
}

Daisy Fancourt ${ }^{\mathrm{a}^{*}}$

${ }^{a}$ Department of Behavioural Science and Health, University College London

*Corresponding author: $\underline{\text { d.fancourt@ucl.ac.uk }}$

\section{The arts and public health}

There is growing research demonstrating the impact of the arts and culture on health and wellbeing. At a population level, research suggests that people who engage in the arts have a lower relative risk of dying prematurely and are statistically more likely to report good health. ${ }^{1,2}$ In England alone, the arts, museums and heritage sites are anticipated to save the NHS around $£ 700$ million per year through reducing GP visits and use of mental health services. ${ }^{3}$ At an individual level, research is now showing the impact of the arts on biological, psychological, social and behavioural aspects of health across the lifespan. ${ }^{4}$

In light of this growing evidence base, a number of organisations have been set up internationally to support the development of the field. Taking the UK as an example, the National Alliance for Arts, Health and Wellbeing launched in 2012 to encourage the use of the arts by health and social care providers and encourage best quality practice. The Royal Society for Public Health founded a Special Interest Group in 2015 to share current research, organise conferences, and influence government policy as a professional body. And in 2014 an All Partly Parliamentary Group (APPG) for Arts, Health and Wellbeing was established to engage cross-bench membership of politicians.

\section{The importance of research}

The APPG has just finished a political inquiry, which has foregrounded the importance of research. ${ }^{5}$ One of the ten recommendations is for research councils in the UK to consider an interdisciplinary, cross-council research funding initiative for the field. Such funding would undoubtedly advance the evidence base. However, an important consideration is whether there are enough researchers to lead such work.

Currently, there are limited opportunities globally for those interested in the field to undertake taught degree courses in Arts and Health. There is some evidence that this is changing, both through new courses emerging and a growing number of funded PhD studentships. Further, the APPG report has also recommended that accredited modules on arts, health and wellbeing be included in the education of clinicians, public health specialists, other health professionals, and those undertaking arts-based education. So perhaps we will shortly see many more training opportunities being developed. Nevertheless, for now the gap remains.

Consequently, there is only a limited pool of researchers who have formal training in working in the field and a lack of clear pathways for students to careers in Arts and Health research. In order to realise the ambitions that are being set out within the field, there is a clear need to identify more researchers interested in working in Arts and Health, provide opportunities for them to learn more about the field and provide bespoke training to supplement existing knowledge and experience and equip them to lead future research projects.

\section{Arts Health ECRN}

In recognition of this need, in March 2017, a new Arts Health Early Career Research Network (ECRN) was launched. Funded by the British Academy, this network brings together early career researchers working on projects that lie at the intersection of the arts, humanities, health and medicine. It is free to join and open to students, researchers who are within 8 years of receiving their $\mathrm{PhD}$, and early career professionals working in research or evaluation. The network aims to be an enabling space, with early career researchers setting its agenda and contributing to its running. Nevertheless, to provide guidance, activity is carried out under the ECRN's three main aims: link, learn and lead. 
'Link' is about linking together early career researchers across the UK and internationally through facilitating social events and networking opportunities to encourage collaboration. The network has a growing number of 'area representatives' who are responsible for identifying potential new members in the region or country they represent, collating details about already existing networking opportunities for publicising through the ECRN website, and coordinating bespoke events on behalf of the ECRN. The network's blog is a way for those who are unable to attend to maintain their link to these events.

Learn

'Learn' is about providing resources for early career researchers to help them become better acquainted with the field. The ECRN curates an online library of textbooks, taking recommendations each month for new additions with members writing reviews about what each book adds to the field as a whole. There are also free podcasts released every few weeks, which provide bitesize updates on aspects of research, policy and practice.

\section{$\underline{\text { Lead }}$}

Finally, 'lead' is about supporting the training of early career researchers interested in arts and health. The network has launched the first of its own bespoke training opportunities in order to help early career researchers develop their research ideas and design and submit strong funding applications. It also aims to become a hub for advertising other courses and conferences, hopefully enabling a broader pool of interested researchers to engage with the field.

Within the first 10 weeks of launching in late March 2017, the network had over 300 members join from 25 countries across 6 continents, and it is growing daily; over 500 members at the time of writing. Members have cited diverse arts interests in architecture, dance, design, digital arts, film, galleries, literature, museums, music, theatre, visual arts and writing, as well as scientific interests in anthropology, economics, dentistry, disability research, education, epidemiology, geography, history, medical humanities, medicine, neuroscience, nursing, philosophy, psychiatry, psychology, psychoneuroimmunology, public health, sociology and statistics. Many members are already active researching in the field, including carrying out studies in hospitals, hospices, care homes and the community as well as in laboratories and with population databases.

It is anticipated that activity within the network will not only be of interest to researchers but also to clinicians, artists and those involved in policy. So, in support of the field as a whole, the website and resources are freely available to all. However, this network has research at its heart: aiming to identify, nurture and enable the next generation of researchers in this field, and thereby strengthen the research outlook for the future.

For more information, visit www.artshealthecrn.com

\section{Acknowledgements}

The Arts Health ECRN is funded by a British Academy Rising Star Engagement Award held by the author. This work was supported by the Wellcome Trust [205407/Z/16/Z]. The author would like to acknowledge Katey Warran for her pivotal role in establishing the network and the ECRN representatives supporting its development.

\section{References}

1. Johansson, S. E., Konlaan, B. B. \& Bygren, L. O. Sustaining habits of attending cultural events and maintenance of health: a longitudinal study. Health Promot. Int. 16, 229-234 (2001).

2. Bygren, L. O., Konlaan, B. B. \& Johansson, S.-E. Attendance at cultural events, reading books or periodicals, and making music or singing in a choir as determinants for survival: Swedish interview survey of living conditions. BMJ 313, 1577-1580 (1996).

3. Fujiwara, D., Kudrna, L., Cornwall, T., Laffan, K. \& Dolan, P. Further analysis to value the health and educational benefits of sport and culture. (DCMS, 2015).

4. Fancourt, D. Arts in Health: Designing and researching interventions. (Oxford University Press, 2017).

5. Creative Health: The Arts for Health and Wellbeing. (All Party Parliamentary Group on Arts, Health and Wellbeing, 2017). 